\title{
A Noble Technique for Digital Image Watermarking Authentication Using Fuzzy Mean Clustering
}

\author{
Rohit Singh ${ }^{1}$, Pancholi Bhavna K. ${ }^{2}$ \\ ${ }^{1,2}$ Electrical Department, Faculty of Technology and Engineering, \\ Maharaja Sayajirao University of Baroda (MSU), Vadodara, Gujarat, India
}

\begin{abstract}
As we all know that in this modern era of technology, digitalization and the internet are very convenient and useful for industrial progress and even for personal users but the main issue is with the shortcomings of security. For instant, private contents are difficult to protect and fake versions of image and videos can be produced. To avoid this suffering digital watermarking provides effective solutions with high level of security. The following work presents a similar but effective image watermarking technique which uses a noble method of fuzzy mean clustering to secure the original contents with proper authentication of a copyright which cannot be easily duplicated. This method shows experimentally that the original image is highly robust against any attacks and even the image degradation is negligibly low. In this proposed method, we don't have to select the embedded bits manually but according to image properties it dynamically selects the embedding bits. Due to this unique feature the original image posses' high security and makes the document immune to attacks.
\end{abstract}

Keywords: Image Watermarking, Authentication, Discrete Cosine Transform, Discrete Wavelet transform, Fuzzy mean Clustering

\section{Introduction}

In the recent years, it becomes a need to create, transmit and distribute digital data over widespread multimedia technology by mean of World Wide Web. Hence the copyright protection has become essential to avoid piracy. Digital image watermarking is the essential mechanism for the ownership authentication.

Image processing is the process of inserting hidden information in the original image by introducing modifications with minimum perception distortion to the image. Some important factors which are to be taken care are - Robustness, Perceptual transparency, Capacity and blind watermarking. Based on robustness of watermarks are classified in three categories. Robust watermark, in which the original contents are totally secure from any attacks; second is fragile watermarks in which original image are prone to attacks and intruders can easily make the changes in the original file whereas the third is the semi fragile watermark in which image are only effected by some specific attacks, Uneven object. .

Basically while working with image modification or image processing we have to choose whether to go with modification in the intensity value of luminance in spatial domain or to change the image coefficient in a frequency domain. We will not go in detail but takes the overview that how frequency domain transformation can be done in image processing and why it is so useful method. Commonly frequency domain transformation includes the Discrete Wavelet Transform and Discrete Cosine Transform.

\section{(A) Discrete Wavelet Transform}

Wavelets are the mathematical functions that cutup data into different frequency components and then study each component with a resolution matched to a scale. In this transformation the host image is subdivided into four different frequency level sub images as LL, HL, LH, and HH. Applying DWT to host image for decompose it into four non-overlapping mutliresolution coefficient sets of an image.

$$
\begin{gathered}
W_{L L}^{J}=\sum_{x=0}^{N-1 N-1} \sum_{y=0}^{N-1} g(x) g(y) W_{L L}^{J-1}(2 u-x)(2 v-y) \\
W_{L H}^{J}=\sum_{x=0}^{N-1 N-1} \sum_{y=0}^{N} g(x) h(y) W_{L L}^{J-1}(2 u-x)(2 v-y) \\
W_{H L}^{J}=\sum_{x=0 y=0}^{N-1 N-1} h(x) g(y) W_{L L}^{J-1}(2 u-x)(2 v-y) \\
W_{H H}^{J}=\sum_{x=0}^{N-1 N-1} \sum_{x=0}^{N} h(x) h(y) W_{L L}^{J-1}(2 u-x)(2 v-y)
\end{gathered}
$$

Where $J$ is the level of the 2-D DWT, $g(n)$ and $h(n)$ are the impulse and $\mathrm{W}_{\mathrm{LL}}=\mathrm{W}(\mathrm{u}, \mathrm{v})$ is the original image.

\begin{tabular}{|c|c|}
\hline LL & LH \\
\hline HL & HH \\
\hline
\end{tabular}

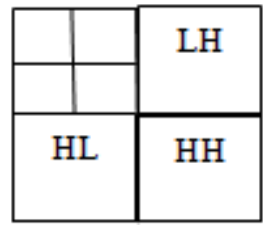

Figure 1

\section{(B) Discrete Cosine Transform:}

This transformation is based on the fact that energy of the most image is concentrated at one place that is the near to origin. This property of image is known as Energy Compaction Property. Apply DCT on each block of the chosen coefficient set WHL. The DCT can be defined by the following equation:

$$
F(u, v)=\sum_{x=0}^{N-1 N-1} \sum g(x, y, u, v)
$$

Where the kernel is given by following equation

$$
g(x, y, u, v)=\alpha(u) \alpha(v) \cos \left[\frac{(2 x+1) u \pi}{2 N}\right] \cos \left[\frac{(2 y+1) v \pi}{2 N}\right]
$$




\section{International Journal of Science and Research (IJSR) \\ ISSN (Online): 2319-7064}

Index Copernicus Value (2013): 6.14 | Impact Factor (2015): 6.391

(C) Fuzzy Mean Clustering:

Clustering is one of the most common techniques of divide input data into groups. The major task is to find the similarity of input data, and retrieve some data points representing each group. Data after clustering is called cluster. Then, the total number of data is reduced and stands for clusters.

In mean clustering techniques, each data must be assigned to one cluster explicitly. But in fuzzy clustering analysis constrain is different it allows gradual memberships. It supports the data to deal with more than one cluster at the same time.

\section{Procedure for Watermarking Embedding}

First we take the original image of $\mathrm{N} \times \mathrm{N}$ size having different intensity value of pixels intensity we will make clusters. The clusters are selected by taking the mean intensity of all the pixels and by that mean value we divide the pixels into two clusters i.e. above and below that mean intensity. In this clustering method we will use the fuzzy for intensity value of pixels which exactly matches the mean value, belongs to both group.

Now, again we take out the mean of both the clusters and further divide the cluster in sub-clusters depend upon the requirements to which level of watermarking we needed. As we get the number of clusters and the mean intensity of each cluster the process of embedding the watermark image could be done.

From this we will make a new image matrix (say $b \times \mathrm{b}$ ) of all that pixels of mean intensity value from that original image. The size of the embedding symbol should made to be the size of new generated matrix $(\mathrm{b} \times \mathrm{b})$ and replace that matrix.

Finally, the position of mean intensity values in original image are occupied by the watermark embedded image. This technique of embedding has used the concept of human eye sensitivity which are less perceptual to certain intensity level, so when the changes takes place in that pixels of the image it will be negligibly effect the image.

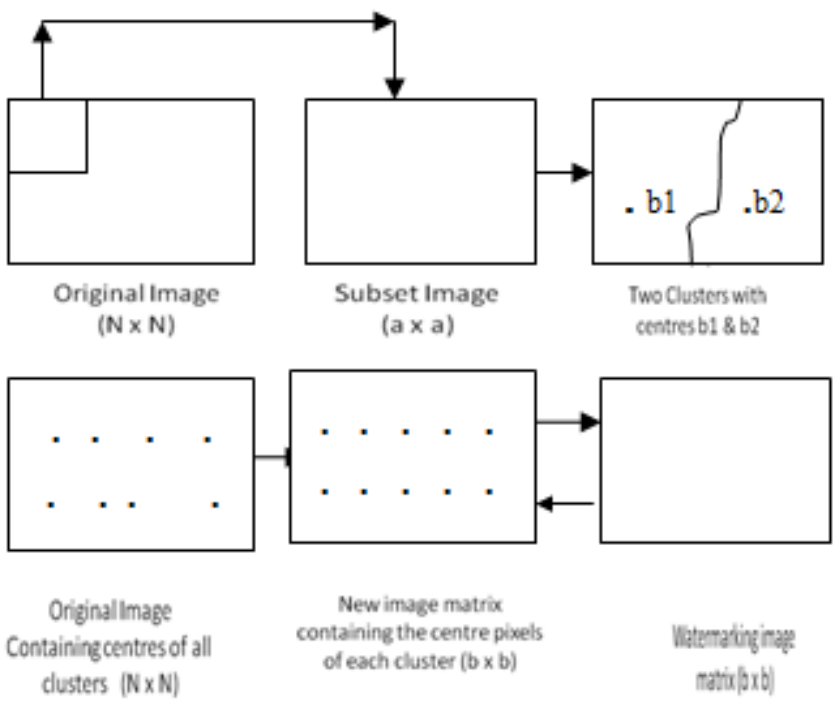

Figure 2

\section{Flow Diagram}

The basic flow diagram for watermark embedding in original image is shown in figure 1. In this work, algorithms are applied on images for embedding image inside it.

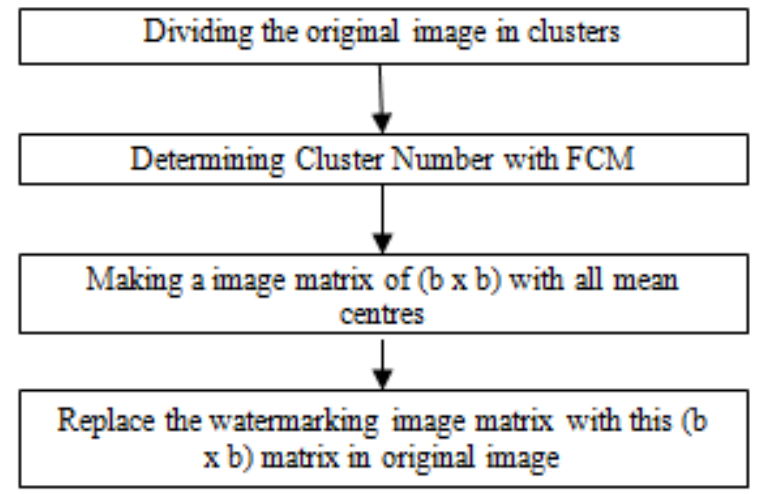

\section{Experimental Results}

To evaluate the effectiveness of the proposed fuzzy mean clustering watermark strategy, we have used two images of different size and of different intensity value as shown below in fig. (a) and fig.(d). These two images are the host images in which watermarked to be performed. Fig.(b) is used as the embedding image which is to be watermarked on the host image. The quality of watermarked image is measured according to PSNR (Peak Signal-to-Noise Ratio) criterion and the degradation is evaluated. By considering the output images we can easily make out the difference in degradation of original image after watermarking. As we can see that fig.(c) is much distorted as compared to fig.(e) and fig.(f) finally giving the more appropriate result. By this method of fuzzy mean clustering the clusters are made on the basis of the intensity levels of the host image and further embedding image pixels are replaced by the this pixel.

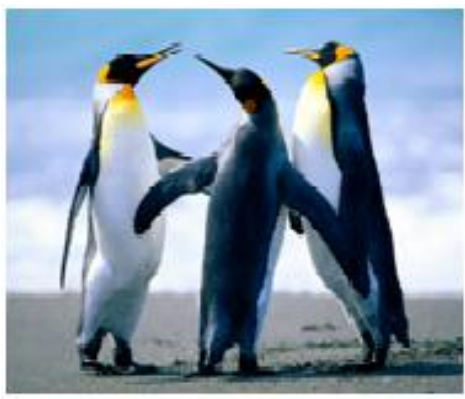

a) Original Input Image

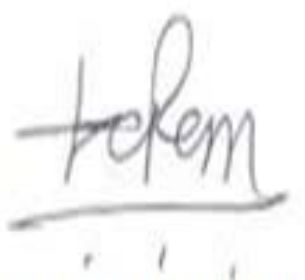

b) Watermarking Image 


\section{International Journal of Science and Research (IJSR) \\ ISSN (Online): 2319-7064}

Index Copernicus Value (2013): 6.14 | Impact Factor (2015): 6.391

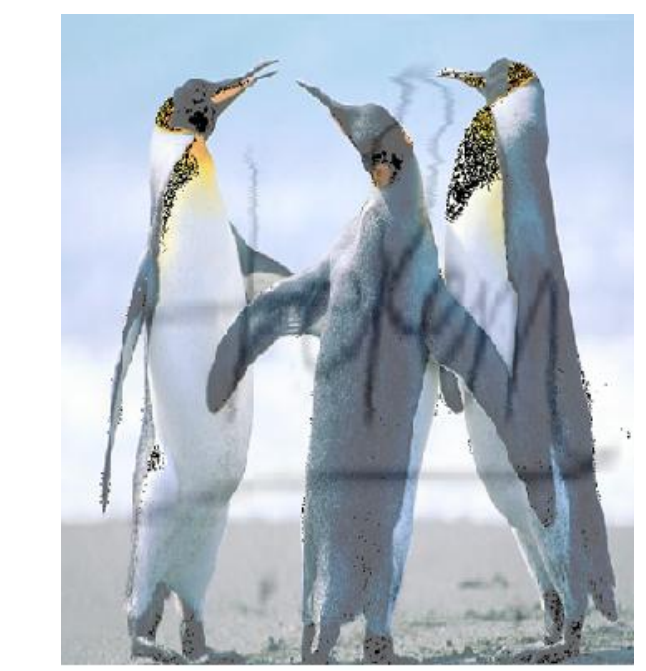

C) Watermarked Image

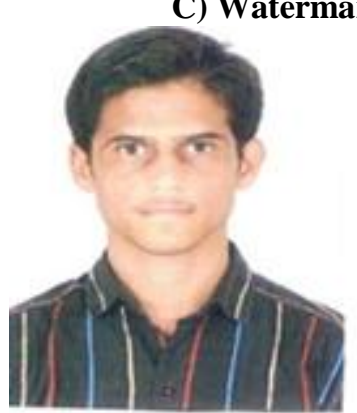

d) Original image

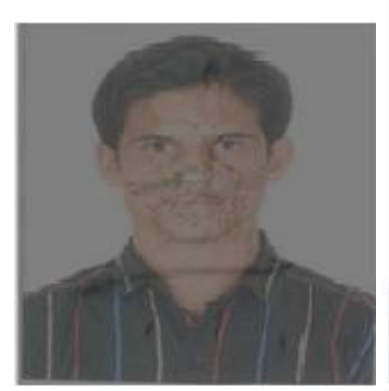

f) Watermarked image I

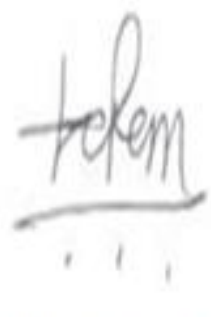

e) Watermarking image

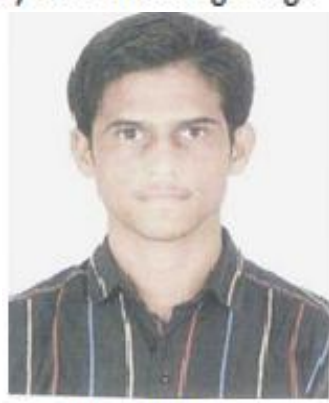

g) Watermaked image II

\section{Advantage of this Work}

- This novel watermarking strategy is presented that does not require manual selection of watermark bits location.

- The number of embedded pixels of clusters differs depending on the intensity characteristics in each image. This significant property possesses higher security.

- This watermarked technique retains maximum quality with very small degradation.

\section{Conclusion \& Future Work}

In this paper, we have described an innovative method of Fuzzy mean clustering for image watermarking embedding to get more secure host image and less distortion of a image. Still a major part of this watermarking scheme involves the watermark extraction from host image which will require the reverse procedure of embedding but in effective way.

For future work, further this method will be tested if it will be effective to a compressed video by using motion vector or by mean intensity.

\section{References}

[1] S. Bhattacharya, T. Chattopadhyay, and A. Pal, "A survey on different video watermarking techniques and comparative analysis with reference to h.264/avc," in IEEE Trans. on Image processing , June 2006, pp.73-78.

[2] Ming-Shing Hsieh, "Perceptual Copyright Protection using multiresolution Wavelet Based Watermarking and Fuzzy Logic," International Journal of Artificial Intelligence \& Application (IJAIA), Vol.1, No.3, July 2010.

[3] Chiou-Ting Hsu and Ja-Ling $\mathrm{Wu}$, "Multiresolution Watermarking for Digital Images," IEEE trans. Circuits and system II, Vol. 45, No.8, pp.1097-1101, August 1998

[4] Deb kaushik, Md.Sajib Al-Seraj, Md.Moshiual Hoque "Combined DWT - DCT Based Digital Image Watermarking Technique for Copyright Protection," $7^{\text {th }}$ International Conference on Electrical and Computer Engineering, 20-22 Dec,2012,Dhaka,Bangladesh.

[5] T.K.Truong, J.H.Jeng, I.S.Reed and A.Q.Li,"A Fast Encoding Algorithm for Fractal Image Compression Using the DCT Inner Product", IEEE Trans. on IP, vol.9,pp.529-535,Apr. 2000.

[6] C. H. Kung, J. H. Jeng, Y. C. Lee, H. H. Hsiao, and W. S. Cheng, "Video watermarking using motion vector," in 16th IPPR Conference on Computer Vision Graphics and Image Processing (CVGIP 2003), August 2003, pp. 547551.

[7] S. Mabtoul, E. Tbn-Elhaj and D. Aboutajdine, "A blind chaos- basedcomplex wavelet domain image watermarking technique,"International Journal on Computer Science and Network Security, Vol.6, No. 3, March 2006

[8] H. Ren-Junn, K. Chuan-Ho, and C. Rong-Chi, "Watermark in color image," Proceedings of the first International Symposium on Cyber Worlds, pp. 225229,2002 .

[9] Chang, and Zhang, "Fuzzy-ART based adaptive digital watermarking scheme," iEEE Trans. on Circuits and Systems for Video Technology Vol. 25, No.1, pp.65-81, 2005. 